\title{
O PERFIL DOS CANDIDATOS AO CURSO TÉCNICO DE ENFERMAGEM DE UMA ESCOLA PARTICULAR DA CIDADE DE SÃO PAULO
}

\author{
THE TECHNICAL NURSES PROFILE AT A PRIVATE SCHOOL OF NURSING IN \\ SAO PAULO CITY
}

\author{
Marcos Antonio da Eira \\ Frias* Regina Toshie \\ Takahashi**
}

FRIAS, M.A.E.; TAKAHASHI, R.T. O perfil dos candidatos ao curso técnico de enfermagem de uma escola particular da cidade de São Paulo. Rev.Esc.Enf.USP, v.34, n.2, p. 309-16, set. 2000.

\section{RESUMO}

Este estudo teve como objetivo levantar o perfil dos candidatos ao curso técnico de enfermagem de uma Escola de Enfermagem Particular da cidade de São Paulo. A população consta de indivíduos adultos jovens; com idade média de 31,09 anos; com renda familiar de 9,65 salários minimos; solteiro; predominância do sexo feminino; 12,5\% deles com formação escolar superior incompleto ou completo; 43,8\% tem filhos, cujas idades variam entre 6 e 13 anos. Os candidatos apresentam afinidade para trabalhar com pacientes graves e sem afinidade para assistência pediátrica. Do trabalho em oncologia, o aprendizado pessoal é fator positivo em oposição ao sofrimento pessoal e do paciente, que é considerado aspecto negativo.

PALAVRAS-CHAVE: Perfil do técnico de enfermagem. Ensino médio de enfermagem.

\section{ABSTRACT}

The result showed the candidate's profile as they are adult; with age mindle of 31,09 years old; familiare income are 9,65 minimun salary; single; predominant female; 12,5\% with university course complete or incomplete; $43,8 \%$ have a children. The age of a children vary of 6-13 years old. The candidates refer afinity to work with patient of major risk and absence of afinity to pediatric patient. The work in oncology, demonstrated the positive way the personal learning and the negative, the personal and patient suffering.

KEYWORDS: Nursing education. Nurses.

\section{INTRODUÇÃO}

Com a promulgação da Lei de Diretrizes e Bases da Educação Nacional (L.D.B.) 9394/96 (BRASIL, 1996), os cursos Técnicos entraram em evidência, uma vez que estes foram totalmente desvinculados do Ensino Médio.

Na vigência da L.D.B. (5692/71), tais cursos eram previstos com a denominação de Curso de Qualificação Profissional IV (Q.P. IV).(BRASIL, 1971)

NARCHI (1994), baseou seus estudos na L.D.B $5692 / 71$ e afirmou que com o aprimoramento advindo da legislação normativa subsequente, e em vigor, - Resolução CFE n $07 / 77$ e Deliberação CEE $\mathrm{n}^{\circ}$ 25/77 (BRASIL, 1997; SÃO PAULO, 1977) - a habilitação plena de técnico de enfermagem pode ser alcançada por três tipos de cursos: o de ensino regular de $2^{\circ}$ Grau com conteúdo profissionalizante e duração de quatro anos; o de ensino supletivo de formação profissional realizado após a conclusão do $2^{\circ}$ Grau, com duração média de dezoito meses; e, o de ensino supletivo ou complementação, oferecido ao auxiliar de enfermagem que tenha completado o $2^{\circ}$ Grau, com duração de aproximadamente seis meses.

Enfermeiro, Diretor de Escola de Nível Médio, Mestrando em Enfermagem, Escola de Enfermagem da USP - área de concentração Administração dos Serviços de Enfermagem.

** enfermeiras Professora Doutora do Departamento de Orientação Profissional da Escola de Enfermagem da USP 
Além do Q.P. IV existiam os Cursos de Qualificação Profissional I, II e III (Q.P. I, Q.P. II e Q.P. III), que podiam ser cursados com o Ensino Fundamental (antigo $1^{\circ} \mathrm{Grau}$ ), incompleto ou completo. Dentro da classificação Q.P. III encontravam-se os Cursos de Auxiliar de Enfermagem.

Apesar das possibilidades, de formação do técnico de enfermagem, o maior enfoque na formação de profissionais de nível médio em enfermagem, sempre foi a formação de auxiliares de enfermagem, uma vez que para a formação deste profissional o grau de escolarização exigida era somente, o Ensino Fundamental (antigo $1^{\circ}$ Grau) completo.

Com a L.D.B. 9394/96 (BRASIL, 1996) deixam de existir as denominações de curso de Qualificação Profissional I, II, III e IV, definidos pelos artigos 18 e 19 da Deliberação C.E.E. 23/83, passando a serem chamadas de acordo com o novo ordenamento legal, de Qualificação Profissional Básica, não sujeita a regulamentações curriculares; Qualificação Profissional de Auxiliar Técnico, que não é propriamente uma Habilitação Profissional e a Habilitação Profissional de Técnico de Nível Médio (SÃO PAULO, 1997).

No nosso entender, nos últimos anos com a maior oferta de cursos supletivos de Ensino Fundamental e Médio, a população brasileira em especial das grandes capitais passou a ter maior facilidade de acesso à escolarização, este fato somado as inovações trazidas pela LDB 9394/96 (BRASIL, 1996), no que se refere à desvinculação dos cursos técnicos do Ensino Médio e a ênfase dada pelo Conselho Regional de Enfermagem de São Paulo ao que prevê a legislação que regulamenta o exercício profissional, conduziu os auxiliares de enfermagem a buscarem um aprimoramento de sua formação na área de enfermagem através dos cursos de aproveitamento de estudos (antiga complementação), sendo assim a soma desses fatores vem gradativamente conduzindo à mudança do perfil dos profissionais de nível médio de enfermagem na cidade de São Paulo.

\section{OBJETIVO}

Levantar o perfil dos candidatos ao curso técnico de enfermagem de uma Escola de Enfermagem particular na cidade de São Paulo, cujas respostas nortearão a condução do curso, possibilitando uma avaliação diagnóstica.

\section{METODOLOGIA}

Este estudo, do tipo exploratório descritivo, foi realizado na Cidade de São Paulo, em uma Escola de Enfermagem particular, cuja mantenedora é uma instituição filantrópica de ensino e pesquisa na área de oncologia e, além dos cursos de aprimoramento para nutricionistas; residência médica; mestrado e doutorado, mantém uma Escola para formação de profissionais Técnicos de Nivel Médio em enfermagem. Mantém também um Hospital especializado no atendimento de pacientes oncológicos.

A população do estudo consta de 32 (trinta e dois), candidatos ao curso técnico de enfermagem, o qual foi estruturado para uma clientela específica de auxiliares de enfermagem que atuavam no hospital da mantenedora.

Do total de candidatos inscritos, $28(87,5 \%)$ pertenciam ao hospital da mantenedora e 4 $(12,5 \%)$, ao hospital cedente do campo de estágio de enfermagem obstétrica.

A coleta de dados foi feita no período de 23 a 25 de março de 1999, a partir de um questionário (anexo), entregue aos candidatos no ato da inscrição, quando se solicitou o preenchimento. Os dados obtidos foram analisados em número absoluto e percentual.

\section{APRESENTAÇÃO DOS RESULTADOS E ANÁLISE}

Hoje mais do que nunca entendemos a importância de se conhecer o perfil dos candidatos aos cursos técnicos de nível médio. Considerandose as condições sócio-econômicas atuais do País, a disputa cada vez mais acirrada por uma vaga no mercado de trabalho e as mudanças ocorridas em 1986 na legislação que regulamenta o exercício da enfermagem, onde deixam de fazer parte do quadro de profissionais de enfermagem os indivíduos sem formação mínima. 
TABELA 1: DISTRIBUIÇÃO DOS CANDIDATOS AO CURSO TÉCNICO DE ENFERMAGEM QUANTO À IDADE, SÃO PAULO, 1999.

\begin{tabular}{ccc}
$\begin{array}{c}\text { Idade em } \\
\text { anos }\end{array}$ & frequencia & $\%$ \\
$22 \mid----25$ & 7 & 21,9 \\
$25 \mid----28$ & 2 & 6,2 \\
$29 \mid----31$ & 5 & 15,7 \\
$31 \mid----34$ & 8 & 25,0 \\
$34 \mid----37$ & 7 & 21,9 \\
$37 \mid----40$ & 2 & 6,2 \\
$40 \mid---43$ & - & - \\
$43 \mid---46$ & 1 & 3,1 \\
\hline TOTAL & $\mathbf{3 2}$ & $\mathbf{1 0 0}$
\end{tabular}

Dos candidatos, $8(25,0 \%)$ tem idade entre 31 e 34 anos; 7 (21,9\%) entre 22 e 25 anos; 7 (21,9\%) entre 34 e 37 anos; $5(15,7 \%)$ entre 28 e 31 anos.

A maioria dos candidatos são adultos jovens com idade média de 31,09 anos, sendo moradores na cidade de São Paulo e na Grande São Paulo. Mais de $50 \%$ dos candidatos tem idade acima de 31 anos, o que no nosso entender representa outro nível de comportamento e grau de responsabilidade, requerendo maior preparo do professor em relação ao desenvolvimento e uso das estratégias de ensino comunicacionais.

TABELA 2: DISTRIBUIÇÃO DOS CANDIDATOS AO CURSO TÉCNICO DE ENFERMAGEM QUANTO À RENDA FAMILIAR, SÃO PAULO, 1999.

\begin{tabular}{ccccc}
\hline \multicolumn{3}{c}{ Salários minimos } & Freqüência & $\%$ \\
\hline 4,6 & -- & 7,1 & 6 & $\mathbf{2 1 , 9}$ \\
7,1 & -- & 11 & $\mathbf{6 , 2}$ \\
9,5 & 9,5 & 1 & $\mathbf{1 5 , 7}$ \\
12,0 & $-12,0$ & 5 & $\mathbf{2 5 , 0}$ \\
$14,4 \quad-$ & 14,4 & 3 & $\mathbf{2 1 , 9}$ \\
\hline \multicolumn{2}{c}{ TOTAL } & 16,9 & $\mathbf{2 6}$ & $\mathbf{1 0 0 * *}$ \\
\hline
\end{tabular}

** $100 \%$ equivale a 26 candidatos respondentes
Da população total em estudo, $11(42,3 \%)$ tem renda mensal entre 7,1 e 9,5 salários mínimos; 6 $(23,1 \%)$ entre 4,6 e 7,1 salários mínimos; 5 (19,02\%) entre 12,0 e 14,4 salários mínimos; $3(11,5 \%)$ entre 14,4 e 16,9 salários mínimos; 1 (3,90\%) entre 9,5 e 12,0 salários mínimos e $6(18,75 \%)$ candidatos deixaram a resposta em branco.

Em relação à renda familiar, os respondentes tem uma renda média equivalente a aproximadamente 9,6 salários minimos, confirmando dados obtidos por MOROMIZATO; BAGNATTO (1996), que em seu estudo apresenta dados entre 6 e 11 salários mínimos e PORFÍRIO et al. (1992), que apresenta dados entre 5 e 10 salários mínimos.

TABELA 3: DISTRIBUIÇÃO DOS CANDIDATOS AO CURSO TÉCNICO DE ENFERMAGEM QUANTO AO ESTADO CIVIL, SÃO PAULO, 1999.

\begin{tabular}{lcc}
\multicolumn{1}{c}{ Estado Civil } & Freqüência & $\%$ \\
\hline SOLTEIRO & 17 & 53,12 \\
CASADO & 13 & 40,62 \\
DIVORCIADO & 01 & 3,13 \\
SEPARADO & 01 & 3,13 \\
\hline TOTAL & $\mathbf{3 2}$ & $\mathbf{1 0 0}$
\end{tabular}

Dos candidatos, 17 (53,12\%) são solteiros, 13 $(40,62 \%)$ são casados. Considerando-se a idade média dos candidatos, o percentual de solteiros é bastante elevado, o que nos permite pensar num provável fator de mudança do perfil da sociedade, uma vez que a maioria dos candidatos é do sexo feminino e que na nossa sociedade as mulheres de uma maneira geral casavam-se muito mais cedo. O maior percentil solteiro, também é citado por VAL (1999) compreendendo $60 \%$ da população estudada (Escola B) e PORFÍRIO et al. (1992) $52,68 \%$.

TABELA 4: DISTRIBUIÇÃO DOS CANDIDATOS AO CURSO TÉCNICO DE ENFERMAGEM QUANTO AO SEXO, SÃO PAULO, 1999.

\begin{tabular}{lcc}
\hline \multicolumn{1}{c}{ Sexo } & Freqüência & \% \\
\hline FEMININO & 23 & 71,87 \\
MASCULINO & 9 & 28,13 \\
\hline TOTAL & $\mathbf{3 2}$ & $\mathbf{1 0 0}$ \\
\hline
\end{tabular}

\footnotetext{
* Salário mínimo de referência em São Paulo (dezembro/1999), R\$ 136,00 (Cento e trinta e seis Reais).
} 
Encontramos o maior percentual dos candidatos $23(71,87 \%)$ é para o sexo feminino e 9 $(28,13 \%)$ para o sexo masculino, confirmando dados de VAL (1999), Escola A $72 \%$ para o sexo feminino, $28 \%$ para o sexo masculino e Escola B $83 \%$ para o sexo feminino, $17 \%$ para o sexo masculino; CARVALHO et al. (1996), 90\% para o sexo feminino, $10 \%$ para o sexo masculino; SILVA (1996), não cita os percentuais, somente faz referência a "maior predominância do sexo feminino"; ANTUNES (1995), $87 \%$ para o sexo feminino, $13 \%$ para o sexo masculino e PORFÍRIO et al. (1992), 84,23\% para o sexo feminino, $15,46 \%$ para o sexo masculino. O predomínio das mulheres no campo da enfermagem ainda é uma realidade, neste estudo apresenta-se numa proporção aproximada de três para um; VIEIRA (1992), refere que nos últimos anos, alguns postos de trabalho de enfermagem passam a ser ocupados pelo sexo masculino sendo que no Estado do Rio de Janeiro, o maior percentual de participação masculina em 1983 era entre os técnicos de enfermagem $15,1 \%$.

TABELA 5: DISTRIBUIÇÃO DOS CANDIDATOS AO CURSO TÉCNICO DE ENFERMAGEM QUANTO A ESCOLARIDADE, SÃO PAULO, 1999.

\begin{tabular}{lcc}
\hline \multicolumn{1}{c}{ Escolaridade } & Freqüência & $\%$ \\
\hline ENSINO MÉDIO & 28 & 87,5 \\
SUPERIOR INCOMPLETO & 03 & 9,4 \\
SUPERIOR COMPLETO & 01 & 3,1 \\
\hline TOTAL & 32 & 100 \\
\hline
\end{tabular}

O Curso de Técnico de Enfermagem, tem como pré-requisito de escolaridade, a conclusão do Ensino Médio.

O que nos chama a atenção neste item, é o fato de $03(9,4 \%)$, dos candidatos referirem possuir curso superior incompleto e $01(3,1 \%)$ curso superior completo. Dos candidatos que referem curso superior incompleto, 01 é de enfermagem, 01 teologia e 01 filosofia; já o candidato com curso superior completo se refere a teologia. Dos autores referenciados, 05 (cinco) citam alunos que possuem curso superior completo ou incompleto. Esses dados levantados por VAL (1999), representam 3\% de sua população; CARVALHO(1996), 0,4\% concluíram ou estão concluindo o curso superior; ANTUNES(1995), 1,6\%; PORFÍRIO(1992), 5,36\% curso superior completo e $1,58 \%$ curso superior incompleto e VIEIRA(1992), $17,7 \%$ apresentam $3^{\circ}$ Grau completo.
TABELA 6: DISTRIBUIÇÃO DOS CANDIDATOS AO CURSO TÉCNICO DE ENFERMAGEM QUANTO A ARRIMO DE FAMÍLIA, SÃO PAULO, 1999.

\begin{tabular}{lcc}
\hline \multicolumn{1}{c}{ Arrimo } & Freqüência & $\%$ \\
\hline SIM & 14 & 44,0 \\
NÃO & 8 & 25,0 \\
EM BRANCO & 10 & 31 \\
\hline TOTAL & $\mathbf{3 2}$ & $\mathbf{1 0 0}$ \\
\hline
\end{tabular}

Entre os candidatos, 14 (44\%) são arrimo de família, quando associados aos dados sexo com predominância feminino $23(71,84 \%)$ e estado civil casado $13(40,62 \%)$, nos leva a pensar na situação das mulheres arcarem com o sustento da casa. Ainda nesta questão recebemos $10(31,0 \%)$ respostas em branco, o que nos leva a supor que os respondentes não entenderam o significado da palavra arrimo. VAL (1999), em sua pesquisa levantou este dado com um percentual de $15,46 \%$, sendo este bastante inferior ao que obtivemos.

TABELA 7: DISTRIBUIÇÃO DOS CANDIDATOS AO CURSO TÉCNICO DE ENFERMAGEM QUANTO AO NÚMERO DE FILHOS, SÃO PAULO, 1999.

\begin{tabular}{ccc}
\hline Número de filhos & Freqüência & $\%$ \\
\hline NÃO TEM & 11 & 34,4 \\
01 & 3 & 9,4 \\
02 & 8 & 25,0 \\
03 & 3 & 9,4 \\
EM BRANCO & 7 & 21,8 \\
\hline TOTAL & $\mathbf{3 2}$ & $\mathbf{1 0 0}$ \\
\hline
\end{tabular}

Entre os candidatos $11(34,4 \%)$ não tem filhos, dos candidatos que referem ter filhos, $8(25,0 \%)$ tem dois, $3(9,4 \%)$ tem um, $3(9,4 \%)$ tem três. Obtivemos ainda $7(21,8 \%)$ respostas em branco. 
TABELA 8: DISTRIBUIÇÃO DOS CANDIDATOS AO CURSO TÉCNICO DE ENFERMAGEM QUANTO A IDADE DOS FILHOS, SÃO PAULO, 1999.

\begin{tabular}{ccc}
\hline Idade dos filhos & Freqüência & \% \\
\hline 2 meses a um ano & 4 & 14,29 \\
2 a 5 anos & 2 & 7,14 \\
6 a 9 anos & 9 & 32,14 \\
10 a 13 anos & 7 & 25,00 \\
14 a 17 anos & 6 & 21,43 \\
\hline Total & $\mathbf{2 8}$ & $\mathbf{1 0 0}$ \\
\hline
\end{tabular}

* 100 \% corresponde ao número total de filhos

Neste item obtivemos 9 (32,14\%) filhos com idade entre 6 a 9 anos, 7 (25,00\%) filhos com idade entre 10 a 13 anos, $6(21,43 \%)$ filhos com idade entre 14 a 17 anos, 4 (14,29\%) filhos com idade entre 2 meses a 1 ano e $2(7,14 \%)$ filhos com idade entre 2 a 5 anos. Sendo a maior freqüência entre os 6 e 13 anos.

Consideramos importante levantar este dado, uma vez que é sabido que a predominância de mulheres nos cursos de enfermagem é uma realidade, e o fator idade dos filhos, pode por vezes interferir na freqüência das alunas às aulas. A nosso ver, este não é um fator negativo, mas sim, um fator importante que deve ser considerado e avaliado com ponderação pelos docentes que trabalham na educação de adultos. Por tradição a mulher é responsável pelo cuidado e educação dos filhos, e por vezes poderá estar ausente ou necessitar ausentar-se das aulas. Não queremos aqui justificar todas as ausências, mas sim ressaltar que este é um dos vários fatores que permeiam a educação de adultos, os quais a diferenciam do trabalho desenvolvido com crianças, adolescentes e jovens.

TABELA 9: DISTRIBUIÇÃO DOS CANDIDATOS AO CURSO TÉCNICO DE ENFERMAGEM QUANTO A PROBLEMA DE SAÚDE, SÃO PAULO, 1999.

\begin{tabular}{ccc}
\hline Problemas & Frequencias & \% \\
\hline Não & 26 & 81,25 \\
Sim & 1 & 3,12 \\
Em Branco & 5 & 15,63 \\
\hline Total & $\mathbf{3 2}$ & $\mathbf{1 0 0}$ \\
\hline
\end{tabular}

Em relação a problemas de saúde $26(81,25 \%)$ não tem, $1(3,12 \%)$ é hipertenso, $5(15,63 \%)$ deixaram em branco.

TABELA 10: DISTRIBUIÇÃO DOS CANDIDATOS AO CURSO TÉCNICO DE ENFERMAGEM QUANTO A ÁREA DE AFINIDADE, SÃO PAULO, 1999.

\begin{tabular}{ccc}
\hline Area & Freqüência & \% \\
\hline U.T.I. & 14 & 36,84 \\
ENFERMARIA & 6 & 15,78 \\
SEMI-INTENSIVA & 4 & 10,53 \\
PRONTO SOCORRO & 4 & 10,53 \\
PEDIATRIA & 2 & 5,26 \\
AMBULATÓRIO & 1 & 2,63 \\
OUTROS & 7 & 18,42 \\
\hline TOTAL & $\mathbf{3 8}$ & $\mathbf{1 0 0 *}$ \\
\hline
\end{tabular}

*100\% equivale ao número de respostas e não de respondentes

Esta questão possibilitou mais de uma resposta, sendo assim 100\% equivale ao número de respostas e não de respondentes. Se somadas as respostas referentes a preferência para setores onde existem pacientes de maior risco de vida [U.T.I $=14$ $(36,84 \%)$; Semi-Intensiva $=4(10,53 \%)$ e Pronto Socorro $=4(10,53 \%)]$, observa-se que uma maior porcentagem com uma somatória de $57,90 \%$, tem preferência por essas unidades. Consideramos este fator relevante, uma vez que o Decreto 94.406/87 (Brasil - 1987), determina como uma das atribuições do técnico de enfermagem, prestar cuidados diretos a pacientes em estado grave. Obtivemos ainda 7 $(18,42 \%)$ respostas que não foram consideradas, por não serem indicativas de área.

TABELA 11: DISTRIBUIÇÃO DOS CANDIDATOS AO CURSO TÉCNICO DE ENFERMAGEM QUANTO A ÁREA SEM AFINIDADE, SÃO PAULO, 1999.

\begin{tabular}{|c|c|c|c|}
\hline \multicolumn{2}{|r|}{ Area } & Freqüência & $\%$ \\
\hline \multicolumn{2}{|c|}{ PEDIATRIA } & 9 & 25,71 \\
\hline \multicolumn{2}{|c|}{ ENFERMARIA } & 6 & 17,16 \\
\hline \multicolumn{2}{|c|}{ U.T.I. } & 5 & 14,29 \\
\hline CENTRO & CIRÚRGICO & 2 & 5,71 \\
\hline PRONTO & SOCORRO & 1 & 2,85 \\
\hline \multicolumn{2}{|l|}{ NÃO TEM } & 4 & 11,42 \\
\hline \multicolumn{2}{|c|}{ EM BRANCO } & 3 & 8,57 \\
\hline \multicolumn{2}{|c|}{ OUTROS } & 5 & 14,29 \\
\hline \multicolumn{2}{|l|}{ TOTAL } & 35 & $100^{*}$ \\
\hline
\end{tabular}

* $100 \%$ equivale ao número de respostas e não de respondentes. 
A questão possibilitou mais de uma resposta, portanto $100 \%$ equivale ao número de respostas e não de respondentes. Destacamos nesta questão 9 $(25,71 \%)$ repostas para falta de afinidade dos candidatos pelo setor de pediatria, seguido por 6 $(17,16 \%)$ candidatos que referem não ter afinidade para trabalhar com pacientes em unidade de internação médica ou cirúrgica (enfermaria), 5 $(14,29 \%)$ U.T.I., $2(5,71 \%)$ centro cirúrgico, 1 (2,85\%) pronto socorro. Obtivemos ainda como resposta 4 $(11,42 \%)$ tem afinidade com tudo, $3(8,57 \%)$ deixaram em branco. Dos respondentes $5(14,29 \%)$ responderam outras áreas. $\mathrm{O}$ fator falta de afinidade para trabalho em pediatria merece destaque, pois a nosso ver, o profissional de enfermagem que atua nesta área, necessita de uma base de conhecimento científico diferenciado como outras áreas específicas. Estes conhecimentos estão diretamente ligados às fases de desenvolvimento da criança, onde a capacidade de comunicação, percepção e entendimento variam muito de acordo com a idade, exigindo do profissional uma grande interação com a criança.

Os candidatos que referem falta de afinidade por pediatria, justificam sua resposta como "apego à criança"; "cuidar de criança requer 'mais' em todos os aspectos"; "sofrimento pessoal por se apegar à criança"; "envolvo-me muito com crianças, mesmo porque tenho duas"; "por ter filhos pequenos associo a doença para meus filhos e isso não traria bom desempenho profissional"; "fico emocionada". DUPAS (1998), num estudo sobre a percepção de enfermeiras referente a assistência prestada a família e a criança portadora de câncer, diz que na percepção dessas enfermeiras, o processo de cuidar inclui o dar ajuda e apoio psicológico e que em algumas situações, elas não tem estrutura, tempo ou conhecimento para agir.

TABELA 12: DISTRIBUIÇÃO DOS CANDIDATOS AO CURSO TÉCNICO DE ENFERMAGEM QUANTO A ATUAÇÃO EM ONCOLOGIA PONTOS POSITIVOS, SÃO PAULO, 1999.

\begin{tabular}{|c|c|c|}
\hline Positivo & Freqüềncia & $\%$ \\
\hline APRENDIZADO PESSOAL & 13 & 33,33 \\
\hline BEM ESTAR (do paciente) & 8 & 20,51 \\
\hline EXPERIÊNCIA PROFISSIONAL & 6 & 15,39 \\
\hline ASSISTÊNCIA (ao paciente) & 5 & 12,82 \\
\hline $\begin{array}{l}\text { EQUILÍBRIO EMOCIONAL } \\
\text { (pessoal) }\end{array}$ & 2 & 5,12 \\
\hline CURA DO PACIENTE & 2 & 5,12 \\
\hline VALOR À VIDA (dar mais) & 1 & 2,57 \\
\hline $\begin{array}{l}\text { QUALIDADE DE VIDA (do } \\
\text { nariente) }\end{array}$ & 1 & 2,57 \\
\hline GRATIFICANTE & 1 & 2,57 \\
\hline TOTAL & 39 & $100 *$ \\
\hline
\end{tabular}

Por ser esta Escola mantida por uma Instituição Oncológica, a ênfase é dada para a formação de profissionais ao atendimento a essa clientela, o que nos motivou a questionar sobre os pontos positivos do trabalho com oncologia. Obtivemos: $13(33,33 \%)$ aprendizado pessoal, 8 $(20,51 \%)$ bem estar do paciente, $6(15,39 \%)$ experiência profissional, $5(12,82 \%)$ assistência (ao paciente), $2(5,12 \%)$ equilíbrio emocional, 2 (5,12\%) cura do paciente, $1(2,57 \%)$ valor à vida (dar mais), $1(2,57 \%)$ qualidade de vida (do paciente) e 1 $(2,57 \%)$ gratificante.

TABELA 13: DISTRIBUIÇÃO DOS CANDIDATOS AO CURSO TÉCNICO DE ENFERMAGEM QUANTO ATUAÇÃO EM ONCOLOGIA - PONTOS NEGATIVOS, SÃO PAULO, 1999.

\begin{tabular}{lcc}
\hline \multicolumn{1}{c}{ Negativo } & Freqüência & \% \\
\hline SOFRIMENTO PESSOAL & 8 & 25,0 \\
SOFRIMENTO DO PACIENTE & 7 & 21,9 \\
IMPOSSIBILIDADE DE CURA & 4 & 12,5 \\
AÇÕES LIMITADAS (pessoal) & 3 & 9,4 \\
EXPOSIÇÃO OCUPACIONAL & 1 & 3,1 \\
NÃO TRABALHA NA AREA & 1 & 3,1 \\
FALTA DE CONHECIMENTO & 1 & 3,1 \\
(científico) & & \\
NENHUM & 3 & 9,4 \\
EM BRANCO & 4 & 12,5 \\
\hline TOTAL & $\mathbf{3 2}$ & $\mathbf{1 0 0}$ \\
\hline
\end{tabular}

VAL (1999), refere que quando se indagou aos alunos sobre algumas qualidades negativas da profissão, entre outras obteve como resposta o risco de contaminação, desgaste físico e envolvimento emocional levando ao estresse.

$\mathrm{Na}$ questão anterior os respondentes referiram como ponto positivo o aprendizado, apesar de apontarem como ponto negativo em primeiro lugar $8(25,00 \%)$ o sofrimento pessoal no atendimento ao paciente oncológico, seguido de 7 $(21,90 \%)$ sofrimento do paciente e $4(12,50 \%)$ a impossibilidade de cura. Ressaltamos este fato, pois não entendemos nem acreditamos no aprendizado com o sofrimento. Acreditamos sim, que o aprendizado tanto formal quanto informal, deve ser prazeroso e levar o aprendiz a querer cada vez mais novos conhecimentos, que o aprendizado na vida laboral se dá nos (poucos ou muitos) momentos de alegria vivenciados pelo profissional e o sofrimento simplesmente condiciona o individuo a algumas atitudes de defesa momentânea. 
DUPAS et ai. (1998), referindo-se a enfermeiras pediátricas, diz que "a interação com a criança e a família ocorre em um contexto de luta e sofrimento internos, tanto físico quanto psicológico cujo resultado pode ser a sobrevivência ou a morte e que esta convivência constante com a morte exige mecanismos de adaptação para que a enfermeira consiga permanecer no serviço".

Acreditamos que por mais que os candidatos tenham afinidade com a área de oncologia, o trabalho torna-se por vezes penoso, fator que merece atenção especial por parte das chefias, visto que este pode ser gerador de licenças ou faltas, contribuindo para elevar $o$ indice de absenteísmo.

\section{CONCLUSÃO}

Os candidatos são adultos-jovens, solteiros, com predominância do sexo feminino, tendo idade entre 31 a 36 anos (ver tabela 1), moradores na cidade de São Paulo e Grande São Paulo. A renda familiar média equivale a aproximadamente 9,6 salários mínimos. Em relação ao grau de escolaridade levantou-se que $12,5 \%$ dos candidatos tem formação de curso superior incompleto ou completo.

Neste estudo levantamos alguns dados que nos chamam a atenção, tais como a formação escolar de nível superior, um grande número de candidatos arrimo de família além da visão dos candidatos em relação aos pontos positivos e negativos da atuação em oncologia.

Dos candidatos que referem ter filhos, a maior freqüência de idade dos filhos, está entre os 6 e 13 anos.

As áreas com afinidade de atuação, são onde existem pacientes de maior risco de vida e pediatria, unidades de internação médica ou cirúrgica, são consideradas áreas sem afinidade.

Em relação aos pontos positivos e negativos do trabalho em oncologia, destacou-se aprendizado pessoal como ponto positivo; sofrimento pessoal e sofrimento do paciente como pontos negativos. Esses fatores negativos devem merecer atenção especial por parte das chefias, no sentido de favorecer um suporte psicológico para os profissionais de nivel médio de enfermagem, já que estes estão em contato direto com o sofrimento do paciente a maior parte do seu período laboral. Esse contato direto com o sofrimento do paciente produz uma carga de estresse que pode ser gerador de um indice de absenteísmo elevado.

\section{REFERENCIAS BIBLIOGRÁFICAS}

ANTUNES, M.J.M. O exame de suplência para qualificação profissional de auxiliares de enfermagem em Minas Gerais. Rev.Bras.Enf., v.48, n.3, p.304-13, 1995.

BRASIL. Leis etc. Lei n. 5692 de 11 de agosto de 1971. Fixa diretrizes e bases para o ensino de $1^{\circ}$ e $2^{\circ}$ graus, e dá outras providências. Lex: Coletânea de Legislação, jul/ set., p. 1114-25, 1971.

BRASIL. Leis. Decreto 94.406, de 08 de junho de 1987. Regulamenta a Lei $7498 / 86$, que dispõe sobre o exercício da enfermagem, e da outras providências. In: CONSELHO REGIONAL DE ENFERMAGEM DE SAO PAULO - documentos básicos de enfermagem: enfermeiros, técnicos, auxiliares, São Paulo, 1997. p. 43-50.

BRASIL. Leis etc. Lei n. 9394 de 20 de dezembro de 1996. Estabelece as diretrizes e bases da educação nacional. Diário Oficial da União. Brasília, 23 dez. 1996. Seção 1, p. 2783341.

CARVALHO, D.V.et al. Força de trabalho de enfermagem de nivel elementar no Municipio de Belo Horizonte. Rev.Bras.Enf., v.49, n.3, p.343-62, 1996.

DUPAS, G.et al. Percepção de enfermeiras de uma instituição hospitalar sobre a assistência prestada à família e à criança portadora de câncer. Rev.Bras.Cancerol., v.44,n.4, p.32734, 1998.

MOROMIZATO, S.S.; BAGNATTO, M.H.S. Caracterização dos alunos do colégio técnico da UNICAMP. In: CONGRESSO BRASILEIRO DE ENFERMAGEM, 48. São Paulo, 1996. Programa. São Paulo, Associação Brasileira de Enfermagem Seção-SP, 1996, p.265. /Resumo/

NARCHI, N.Z. Cursos técnicos de enfermagem: avaliação da estrutura escolar, da operacionalização curricular e da disciplina enfermagem materno-infantil. São Paulo, 1994. 191p. Dissertação (Mestrado) - Escola de Enfermagem, Universidade de São Paulo.

PORFÍRIO, R.M.et al. Perfil sócio-econômico-cultural do estudante de auxiliares de enfermagem de São Paulo-SP. Rev.Bras. Enf., n.45, v.4, p.290-301, 1992 .

SÃO PAULO (Estado). Conselho Estadual de Educação - Câmara de Ensino Médio. Indicação n ${ }^{\circ} 23$ de 17 de dezembro de 1997. A Educação Profissional e a Nova LDB. Diário Oficial do Estado, São Paulo, 20 dez. 1997. Seção 1, p.16-17.

SILVA, G.T.R. da et al. Perfil do ingressante na escola de formação de profissionais de saúde "Sophia Marchetti". In: CONGRESSO BRASILEIRO DE ENFERMAGEM, 48. São Paulo, 1996. Programa. São Paulo, Associação Brasileira de Enfermagem Seção-SP, 1996, p. 287. /Resumo/

VAL, L.F. do et al Caracterização do perfil dos alunos de nível médio - auxiliares de enfermagem. Nursing, v. 2, n.9, p. $17-21,1999$.

VIEIRA, A.L.S. Perfil do nível elementar de enfermagem no Estado do Rio de Janeiro: caracterização da demanda para a profissão. Rev. Bras.Enf., n.45, v.1, p.15-20, 1992. 


\section{ANEXO}

Nome:

Estado civil:

Idade:

Resid

Bairro:

Renda Familiar:

Arrimo de Família ( ) sim

( ) não

Escolaridade: Ensino Médio (2a Grau): em curso ( ) concluído ( )

\section{Superior (3a Grau):}

Número de filhos: Idade dos filhos:

Problemas de saúde.

\section{AFINIDADE PROFISSIONAL}

Área de afinidade: Justificar:

Área sem afinidade:

Justificar:

\section{ATUAÇÃO EM ONCOLOGIA}

Pontos positivos:

Pontos negativos: 